\title{
Photoinduced Schottky Barrier in Photorefractive Materials
}

\author{
Jaime Frejlich \\ Laboratório de Óptica, IFGW, Universidade Estadual de Campinas, Campinas-SP, Brazil \\ Christophe Longeaud \\ Laboratoire de Génie Eléctrique, CNRS, Supelec, Universités Paris VI et XI, Gif sur Yvette, France \\ Jesiel F. Carvalho \\ Instituto de Física, Universidade Federal de Goiás, Goiânia-GO, Brazil
}

(Received 23 October 2009; published 15 March 2010)

\begin{abstract}
We report on the first experimental evidence of a Schottky barrier effect produced by the action of light in an otherwise purely Ohmic contact between a nominally undoped photorefractive titanosillenite $\mathrm{Bi}_{12} \mathrm{TiO}_{20}$ crystal and a transparent conductive $\mathrm{SnO}_{2}$ electrode. The photorefractive crystal is sandwiched between two transparent electrodes and a Schottky barrier is built up in the illuminated crystal-electrode interface under the action of light with photonic energy large enough to excite charge carriers from the Fermi level into the conduction band. The contact remains purely Ohmic under illumination with photonic energy below that of the Fermi gap and the photoinduced barrier almost disappears if the photonic energy is large enough to produce electron-hole pairs.
\end{abstract}

DOI: 10.1103/PhysRevLett.104.116601

PACS numbers: 72.40.+w, 42.70.Nq, 73.30.+y

The Schottky barrier effect is of the highest importance in modern science and technology and is the basis of a large part of modern electronic devices. The reduction of the Schottky barrier by the action of light is a well established fact and has been already reported before, as, for example, in Refs. [1-3], among others.

In this Letter, however, we report on a quite different result, that is the buildup of a potential barrier due to the effect of light at the interface between a photorefractive crystal and a transparent conductive electrode, the photorefractive material being a titanosillenite $\mathrm{Bi}_{12} \mathrm{TiO}_{20}$ (BTO) crystal and the electrode being a $\mathrm{SnO}_{2}$ thin film. This potential barrier behaves as a Schottky barrier and will be named accordingly in the following.

A $1 \mathrm{~mm}$ thick plate of a photorefractive titanosillenite (BTO) crystal was coated with a conductive transparent $\mathrm{SnO}_{2}$ film on both of the larger sides of the crystal and placed in our photoconductivity measurement instrument [4]. All measurements were carried out at room temperature $\left(\approx 22^{\circ} \mathrm{C}\right)$. The illumination was shone perpendicularly to one of the $\mathrm{SnO}_{2}$-coated surfaces that will be called the front electrode in the following whereas the electrode located on the other side will be called the back electrode. A voltage was applied in between the electrodes, the maximum absolute value of it being equal to $200 \mathrm{~V}$. For clarity, when the potential of the front electrode is higher (lower) than that of the back electrode we shall write that the sample is "positively" ("negatively") polarized. The $i_{\mathrm{ph}}$ notation in this Letter means that the (photo)current measurement is directly associated to the light shining the sample as is the present case where the current is measured using a phase-selective frequency-tuned lock-in amplifier which is reference controlled by the modulated signal operating the light source. The plain $i$ notation without subfixes, instead, means that the current is somehow measured without direct instrumental association to the light. The wavelength-resolved photoconductance per unit incident irradiance $\left(i_{\mathrm{ph}} / V\right) / I(0)$ is shown in Fig. 1 for both positively and negatively polarized crystal, where we see that a sensibly higher photocurrent results from a negative polarization. The $i_{\text {ph }}$ data for each wavelength were measured for different voltages from 0 to $200 \mathrm{~V}$ and linearly fitted to obtain the average conductance $i_{\mathrm{ph}} / V$ that, after normalizing by the incident irradiance $I(0)$, are plotted in Fig. 1. Such a procedure allows one to have a general overview of the asymmetric response for different polar-

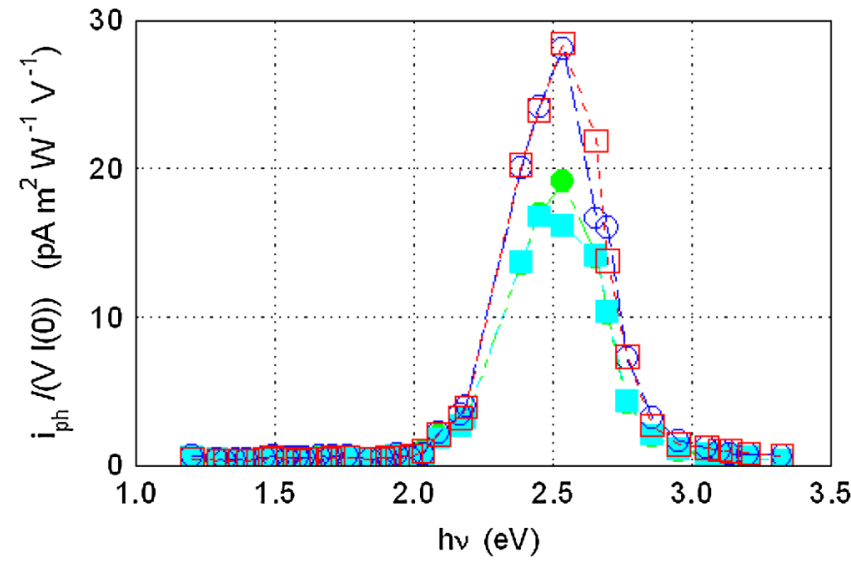

FIG. 1 (color online). Photoconductance per unit incident irradiance (in $\mathrm{pA} \mathrm{m}^{2} \mathrm{~V}^{-1} \mathrm{~W}^{-1}$ ) as a function of the photonic energy $(\mathrm{eV})$ of the illuminating light, for four independent experimental runs with positive (filled circles and squares) and negative (open circles and squares) polarization. 
izations in the whole wavelength range but does not put into evidence the nonlinear responses which are, instead, clearly shown in Fig. 2. Anyway Fig. 1 shows that we may expect different behaviors of the photocurrent versus voltage $i_{\mathrm{ph}}(V)$ curves depending on the energy of the photon impinging the crystal. Indeed, the whole photon energy range of the illumination explored in Fig. 1 can be decomposed into three main photonic energy ranges. A range (rI) for which the photon energy $h \nu$ is lower than the energy gap in between the Fermi level located at $E_{f}$ and the conduction band located at $E_{c}$, typically $h \nu<E_{c}-E_{f}=$ $2.2 \mathrm{eV}$. A second energy range (rII) for which the photon energy is higher than $E_{c}-E_{f}$ but lower than the band gap and a third energy range (rIII) for which the photon energy is larger than the band gap $E_{g} \approx 3.2 \mathrm{eV}$. It is in the range $\mathbf{r I I}$ that we have obtained the more puzzling results. The variations of the photocurrent $\left(i_{\mathrm{ph}}\right.$ in $\left.\mathrm{pA}\right)$ normalized to the incident irradiance $\left(I(0)\right.$ in $\left.\mathrm{W} / \mathrm{m}^{2}\right)$ as a function of the applied voltage for illumination with photonic energies $h \nu=3.1,2.45$, and $2.38 \mathrm{eV}$, corresponding, respectively, to $\lambda=400,506$, and $520 \mathrm{~nm}$, are displayed in Fig. 2. This figure shows that a negative polarization produces a linear Ohmic response whereas with a positive polarization it exhibits a nonlinear response in the lower voltage range with an overall response on the whole range of explored positive voltages lower than for the opposite polarization in agreement with the results of Fig. 1 in the same photon energy range. If light with photonic energy $h \nu=1.9$ or $1.36 \mathrm{eV}$, corresponding, respectively, to $\lambda=$ 651 and $912 \mathrm{~nm}$, is used instead (range $\mathbf{r I}$ ), the response is invariant and always linear, whatever the polarization of the illuminated side of the crystal as displayed in Fig. 3. Finally, in the range rIII with photonic energy of the illumination higher than the band gap, $h \nu=3.32 \mathrm{eV}(\lambda=$ $373 \mathrm{~nm})$, the nonlinear response is no more evident, but still $i_{\mathrm{ph}} / I(0)$ vs voltage curves obtained with a positive polarization exhibit a lower response than for a negative

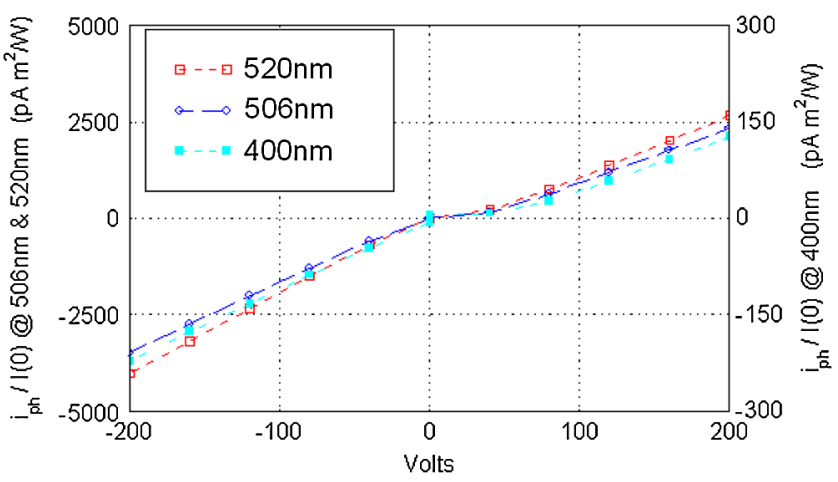

FIG. 2 (color online). Photocurrent per unit incident irradiance (in $\mathrm{pA} \mathrm{m}^{2} / \mathrm{W}$ ) as a function of the applied potential (in V) for 506 and $520 \mathrm{~nm}$ (left-hand axis) and for $400 \mathrm{~nm}$ (right-hand axis). Error bars along the $y$ coordinates are close to the size of the spots on the curves. polarization, as seen in Fig. 4, although in a reduced extent as compared with results in the range rII.

The experiment was repeated on another nominally undoped BTO crystal (labeled BTO-8L), somewhat thicker $(\approx 1.5 \mathrm{~mm})$ than the one we have reported on above, produced at the same laboratory but cut from another boule. The $i_{\mathrm{ph}} / I(0)$ versus voltage curves obtained for this sample at 520 and $400 \mathrm{~nm}$ confirmed our results on the previous sample for the same wavelengths, although exhibiting a less pronounced effect. In addition, another experiment was carried out on this BTO-8L sample, in which the dc current $i$ was measured as a function of the applied dc voltage under CW laser light illumination of $\lambda=514.5 \mathrm{~nm}$ for two largely different (approximately 636 and $13 \mu \mathrm{W} / \mathrm{cm}^{2}$ ) irradiances. The result is shown in Fig. 5 where it is possible to see that for a roughly 50-fold increase in irradiance, the current $i$ increases by $\approx 15$-fold only thus showing a nonlinear relation between $i$ and $I(0)$ thus indicating the different nature of this measurement, as compared to those on $i_{\mathrm{ph}}$, probably because of the presence here of other current components not directly related to photoconductivity. In spite of the lower reliability and the higher noise (compared to photoconductivity $i_{\mathrm{ph}}$ measurements) of data in Fig. 5 it is still possible to detect a nonlinear behavior for positive polarization in the lower voltage range and a lower overall response for positive than for negative polarization, in agreement with the results reported on above for the thinner sample.

It is necessary to point out the presence of a discontinuity of the signal at $V=0$ in Fig. 4 that could be related to an offset of the voltage power source while inverting the signal from one sign to the other. This discontinuity however, is much reduced in Fig. 3 and is not apparent at all in Figs. 2 and 5.

In order to explain our experimental results, we have to consider the optical transitions induced by the photons impinging the crystal. We have thus to consider the distri-

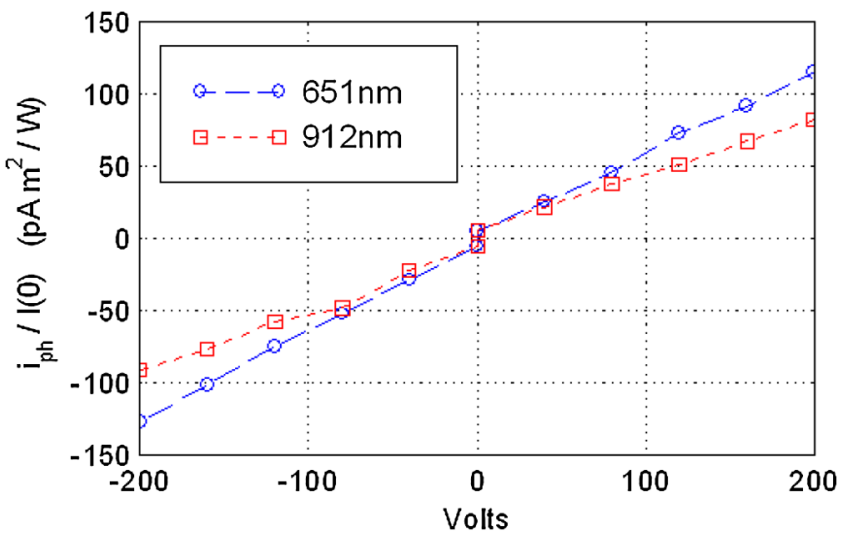

FIG. 3 (color online). Photocurrent per unit incident irradiance (in $\mathrm{pA} \mathrm{m}^{2} / \mathrm{W}$ ) as a function of the applied potential (in $\mathrm{V}$ ) for 912 and $651 \mathrm{~nm}$. Error bars along the $y$ coordinates are close to the size of the spots on the curves. 


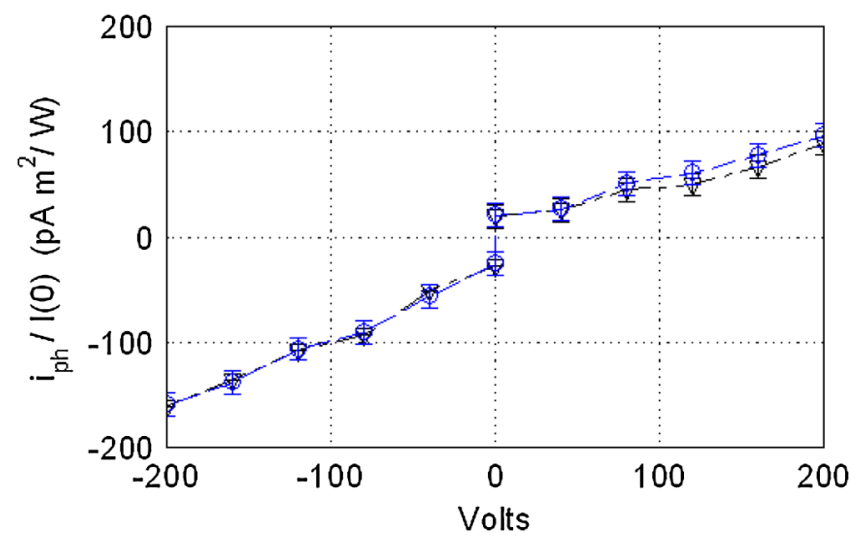

FIG. 4 (color online). Photocurrent per unit incident irradiance (in $\mathrm{pA} \mathrm{m}^{2} / \mathrm{W}$ ) as a function of the applied potential (in V) for $373 \mathrm{~nm}$. Different symbols are for different experimental runs.

bution of gap states in between the two extended state bands. In a previous paper [5] we have proposed, as a result of many experiments performed on BTO crystals, that the density of states (DOS) around and below the Fermi level (known to be at $2.2 \mathrm{eV}$ below the bottom of the conduction band and probably pinned by the $\mathrm{Bi}^{3+}$-pluselectron vacancy defects) was probably made of a continuum of states whereas only few discrete levels exist between $E_{f}$ and $E_{c}$ as schematically described in Fig. 6.

In the range of photon energies $\mathbf{r I}$ the transitions induced from an occupied state towards an empty state are limited. The only possible transitions are from the valence band towards trap states above the Fermi level or from filled gap states located between $E_{v}$ and $E_{f}$ towards empty gap states in between $E_{f}$ and $E_{c}$. Transitions from filled gap states located below $E_{f}$ and the conduction band are impossible. Because only few optical transitions are possible, in this range $\mathbf{r I}$ the absorption is low and the only

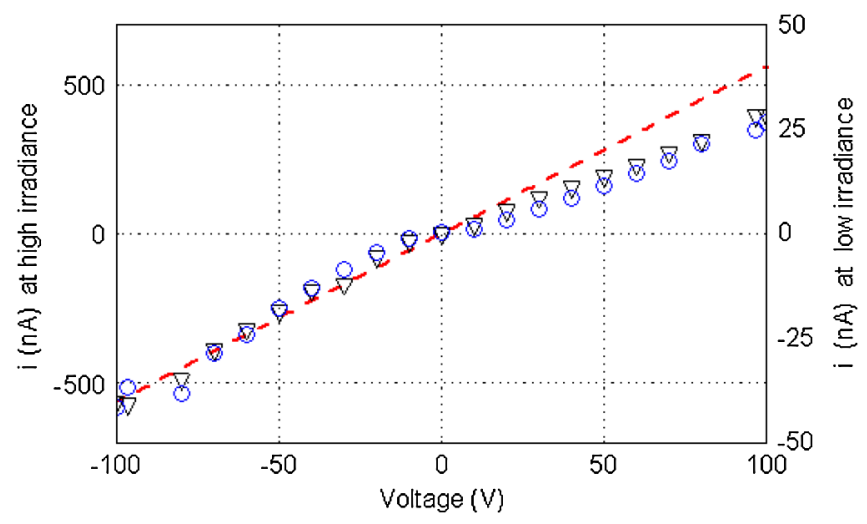

FIG. 5 (color online). Current versus applied voltage for an undoped BTO (labeled BTO-8L) under $\lambda=514.5 \mathrm{~nm}$ light from a laser where $\bigcirc$ are for high irradiance $\left(\approx 636 \mu \mathrm{W} / \mathrm{cm}^{2}\right)$ and are referred to the left-hand ordinate axis whereas $\nabla$ are for weak irradiance $\left(\approx 13 \mu \mathrm{W} / \mathrm{cm}^{2}\right)$ and are referred to the right-hand ordinate axis. The dashed straight symmetric line through $(0,0)$ is just a reference for the eyes. free carriers (holes in the valence band) are generated almost uniformly between the electrodes. For ex., the exponential intensity absorption coefficient $\alpha_{651 \mathrm{~nm}} \approx$ $0.24 \mathrm{~mm}^{-1}$ which means an irradiance $I(0) / 1.4$ at the back crystal (thinner sample) plane for $\lambda=651 \mathrm{~nm}$, with $I(0)$ representing the irradiance at the input plane.

In the range of photon energies rII a new transition takes place from the gap states around and below the Fermi level towards the conduction band generating free electrons in the conduction band. The absorption coefficient increases and the generation of free carriers stops to be uniform through the sample as is the case for $\lambda=506 \mathrm{~nm}$ with $\alpha_{506 \mathrm{~nm}} \approx 1.64 \mathrm{~mm}^{-1}$ which means an irradiance of $I(0) / e$ at a distance of $0.6 \mathrm{~mm}$ from the input crystal plane and $\approx I(0) / 5$ at the back crystal plane. As a result, with no field applied and under steady state illumination, the free carriers tend to diffuse (diffusion length for electrons is estimated to be $L_{D} \approx 0.14 \mu \mathrm{m}$ [6] in this material) towards each electrode. If we take into account the well

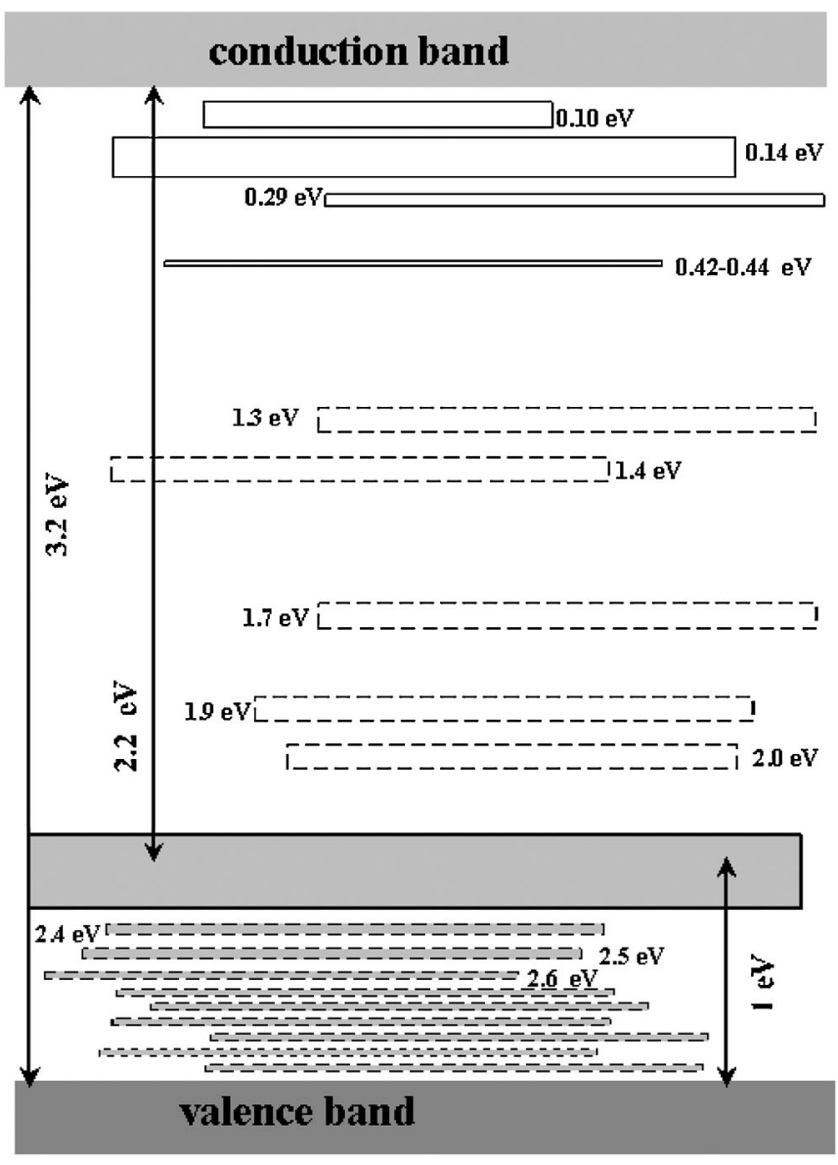

FIG. 6. Schematic representation of localized states in the BTO band gap, with energies measured from the bottom of the CB. Filled electron-donors are in gray and empty levels in white. The density of the states are roughly qualitatively represented by the width of the full-line limited levels. Instead, the width of levels limited by dashed-lines have no relation with their density. The succession of states close to the VB represent the almost continuous localized states in this position with a few ones shown at 2.4, 2.5, and $2.6 \mathrm{eV}$. 
known fact that nominally undoped sillenite crystals exhibit predominantly electron-based photoconductivity in this $\mathbf{r I I}$ range and if we also assume that mobility is higher for electrons than for holes, the former will reach the front electrode, where they recombine, faster than the holes, leaving behind a positive space charge linked to both the free holes and the positively charged gap states that have emitted these electrons. Because of this positive space charge a barrier potential develops close to the front electrode. If the crystal is negatively polarized the diffusion of electrons toward the front electrode is slowed down and the positive space charge is minimized as well as the barrier potential. On the opposite, if the crystal is positively polarized the diffusion of electrons towards the front electrode will be enhanced giving rise to an increased positive space charge. No such a barrier is expected close to the back electrode because of the strongly reduced intensity at the back electrode due to the characteristic high absorption coefficient in this rII range. In the range of photon energies rIII band to band generation takes place. The absorption coefficient increases further on and the free carriers are generated very close to the front electrode. One has still to take account of transitions from gap states located below the Fermi level toward the conduction band in the generation of free electrons but this contribution to the free electron generation is certainly very small compared to the band to band generation. Therefore, the electrical neutrality of the crystal is mainly determined by this band to band generation of carriers that will redistribute into the gap states and the development of a potential barrier should be small if any.

Considering the $i_{\mathrm{ph}}(V)$ curves obtained with different photon energies, in the range $\mathbf{r I}$ no space charge is developed in the crystal and we observe an Ohmic behavior independently of the sign of the polarization. In the range rII a potential barrier develops, the intensity of which is linked to the sign of the polarization and to the light irradiance. With a negatively polarized crystal the barrier is low and the current is not limited by it. With a positively polarized crystal the barrier is high and the electrons have to overcome it. This barrier leads to both a lower current and a non Ohmic $i_{\mathrm{ph}}(V)$ curve. In the range rII I it is still possible to see a larger photocurrent for negative than for positive polarizations probably because of the larger mobility of electrons compared to holes [7] as hole-electron pairs are mainly generated here close to the illuminated front electrode. Almost no nonlinear effects are detected here probably because the space charge that could develop in the states located around and below the Fermi level by optical transitions towards the conduction band is partly masked by the redistribution into the gap states of the free carriers generated by band to band transitions. Hence, the influence of the crystal polarization on the $i_{\mathrm{ph}}(V)$ curves is smaller in the range $\mathbf{r I I I}$ than in the range $\mathbf{r I I}$.
It is important to point out that the light-induced Schottky effect seems to be less evident in the thicker $(1.5 \mathrm{~mm})$ sample as compared to the thinner $(1 \mathrm{~mm})$ one. We believe this fact results because the thicker sample exhibits a much poorer illumination $(\approx I(0) / 12$ for the thicker as compared to $I(0) / 5$ for the thinner sample, for $\lambda=506 \mathrm{~nm}$ ) at the output crystal plane, thus strongly reducing the effective voltage applied in the illuminated region close to the front electrode and thus reducing the effect on the Schottky barrier too.

We have reported on what seems to be the first experimental evidence of a Schottky barrier effect induced by the action of light at the interface between a photorefractive crystal and a transparent conductive electrode. This interface has a perfect Ohmic behavior in the absence of light or under light with photonic energy low enough not to give rise to free charge carriers in the extended states. That is to say that an interface contact can be switched from rectifying to perfectly Ohmic and back by the simple action of light.

We believe that the photoinduced Schottky effect here reported on, is likely to be observed in other photorefractive materials because they characteristically exhibit a large number of photoactive localized states in the forbidden gap that can produce monopolar free charge carriers at photonic energies below that of the band gap. The practical possibilities of this effect however, should require further research to find out the optimal materials, geometries and configuration.

We are grateful to Victor Pellegrini Mammana, Luiz Alberto Castro de Almeida, Iraci da Anunciação Pereira, and Maria Garcez da Silva from the Centro de Tecnologia da Informática Renato Archer (CTI) at Campinas, that kindly prepared the $\mathrm{SnO}_{2}$ transparent conductive electrodes on our photorefractive sample. We also thanks Tatiane Oliveira dos Santos for preparing the sample for the present experiment.

[1] M. Thompson, J. Allison, M. Al-Kaisi, and I. Thomas, Rev. Phys. Appl. 13, 625 (1978).

[2] M. Qin, K. Yao, and Y.C. Liang, J. Appl. Phys. 105, 061624 (2009).

[3] M. C. Newton, S. Firth, and P. A. Warburton, IEEE Trans. Nanotechnol. 7, 20 (2008).

[4] R. Montenegro, N. R. Inocente-Junior, and J. Frejlich, Rev. Sci. Instrum. 77, 043905 (2006).

[5] J. Frejlich, R. Montenegro, N. R. Inocente-Junior, P. V. dos Santos, J. C. Launay, C. Longeaud, and J. F. Carvalho, J. Appl. Phys. 101, 043101 (2007).

[6] I. de Oliveira and J. Frejlich, J. Opt. Soc. Am. B 18, 291 (2001).

[7] R. H. Bube, Photoelectronic Properties of Semiconductors (Cambridge University Press, Cambridge, England, 1992), 1 st ed. 